\title{
Abnormal Responses of Myeloid Progenitor Cells to Granulocyte-Macrophage Colony-stimulating Factor in Human Cyclic Neutropenia
}

\author{
Daniel G. Wright, Vincent F. LaRussa, August J. Salvado, and Robert D. Knight \\ Department of Hematology, Walter Reed Army Institute of Research, Washington, DC 20307-5100
}

\begin{abstract}
Granulocyte-macrophage progenitors (CFU-GM) from four patients with childhood onset cyclic neutropenia demonstrated abnormal in vitro proliferative responses to purified, recombinant human granulocyte-macrophage colony-stimulating factor (rhGM-CSF) when examined in detailed dose-response studies. Marrow aspirate specimens were obtained for these studies from cyclic neutropenia patients (both during neutropenic nadirs and during recovery phases of cycles), from leukemia patients in remission who had received myelosuppressive chemotherapy, and from healthy normal volunteers. Nucleated marrow cells were then isolated by density-gradient centrifugation and cryopreserved to permit studies of CFU-GM from patients and controls to be carried out at the same time and in replicate. Maximum clonal growth of CFU-GM from normal subjects and from individuals recovering from drug-induced myelosuppression was elicited by 20-100 pmol/liter rhGMCSF, and the CSF concentrations that induced half-maximal responses $\left(E D_{50}\right)$ were between 1.0 and $3.0 \mathrm{pmol} / \mathrm{liter}$. In contrast, maximum growth of CFU-GM from the cyclic neutropenia patients required $\geq 1.0 \mathrm{nmol} /$ liter $\mathrm{rhGM-CSF}$ and ED $_{50}$ 's were $>30.0 \mathrm{pmol} / \mathrm{liter}$. These abnormalities in the GM-CSF responsive growth of myeloid progenitors were independent of cycle time and were most apparent with the predominantly neutrophilic 7-d CFU-GM. Moreover, differences in the growth of 14-d CFU-GM could be attributed mostly if not entirely to differences in the generation of neutrophilic colonies. These findings indicate that childhood onset cyclic neutropenia is associated with an underlying disturbance in the GM-CSF responsive growth of myeloid progenitors committed to neutrophilic differentiation.
\end{abstract}

\section{Introduction}

Human cyclic neutropenia (or "cyclic hematopoiesis") is a rare but very distinctive disorder characterized by regular oscillations in blood neutrophil and monocyte counts (1). Neutrophil counts fall to profoundly low levels at regular 3-wk intervals, followed by a recovery phase during which neutro-

Address reprint requests to Dr. D. G. Wright, Department of Hematology, Walter Reed Army Institute of Research, Washington, DC 20307-5100.

Received for publication 8 August 1988 and in revised form 18 November 1988

The Journal of Clinical Investigation, Inc.

Volume 83, April 1989, 1414-1418 phils return to the circulation and may attain normal levels. Monocyte counts also cycle but do so out of phase with neutrophils, usually fluctuating between high normal and distinctly elevated levels. Cyclic neutropenia occurs both as a childhood onset and an adult onset disease $(1,2)$. The latter form of the disease has been shown to respond to low-dose, alternate-day cortico-steroid therapy $(2,3)$ and has been associated with a "benign" lymphoproliferative neoplasm of large granular lymphocytes (2). Childhood onset cyclic neutropenia, on the other hand, appears to be congenital in most cases, is not associated with a lymphoproliferative disorder, and commonly occurs in a familial pattern suggestive of an autosomal dominant mode of inheritance $(1,2)$.

Studies of neutrophil kinetics in both adult and childhood onset cyclic neutropenia have shown that the oscillations of blood neutrophil counts result from abnormalities in the regulation of neutrophil production and not from intermittent destruction or an abnormal utilization of these cells $(1,4)$. Although studies of hematopoietic progenitors in human cyclic neutropenia have been limited, this disease does not appear to be associated with reduced numbers of myeloid colony-forming cells (CFU-GM). ${ }^{1}$ Rather, CFU-GM concentrations in the marrow have been reported to fluctuate between normal and above normal levels with peak levels coinciding with nadirs of neutropenia (5-8).

Several mathematical models of the regulation of myelopoiesis have been based upon the distinctive features of cyclic neutropenia (9-12). These models have predicted that myelopoiesis should be intrinsically susceptible to oscillations if the proliferation and maturation of myeloid progenitor cells is regulated by a balance of positive and negative signals, involving a feedback loop in which regulatory signals are controlled in part by the mature cells (e.g. neutrophils and macrophages) that are generated. Futhermore, these models have provided a basis for the hypothesis that an abnormality in the responsiveness of progenitor cells to competing regulatory signals could provoke cycling (12).

Recently, it has become possible to test this hypothesis by studying the clonal proliferation of myeloid progenitor cells in response to purified myelopoietic growth factors. Not only is there now substantial evidence that both positive and negative myelopoietic regulatory factors exist (13-16), but a number of these factors have been characterized at a molecular level by genetic cloning and expression of biologically active recombinant proteins $(13,14)$. In the studies reported here, we have

1. Abbreviations used in this paper: CFU-GM, granulocyte-macrophage progenitors; GM-CSF, granulocyte-macrophage colony-stimulating factor; $h$, human; $r$, recombinant. 
used purified, recombinant human granulocyte-macrophage colony-stimulating factor (rhGM-CSF) to identify and characterize abnormalities in the GM-CSF responsive growth of myeloid progenitors in childhood onset cyclic neutropenia.

\section{Methods}

Human subjects. Bone marrow aspirate specimens were obtained from four patients (two females, ages 14 and 28, and two males, ages 26 and 37) with well-documented childhood onset cyclic neutropenia, from two male patients with acute leukemia in hematologic remission (ages 24 and 51) who had received high-dose myelosuppressive chemotherapy in post remission induction intensification protocols, and from eight healthy normal volunteers (ages 19-43) after informed consent in accordance with a protocol approved by the Clinical Investigation Committee of the Walter Reed Army Medical Center. All patients with cyclic neutropenia had been followed clinically (by D. Wright) for 6-12 yr and were known to have maintained consistent neutrophil cycles with stable cycle lengths and amplitudes; three of these patients have been described previously (reference 1, patients 1, 5, and 8). Marrow aspirates from these patients were timed to coincide both with the 5-7-d periods during cycles when neutrophil counts were lowest ("nadir") and with days when counts were highest ("recovery"). Marrow specimens from the patients who had received myelosuppressive chemotherapy were obtained 10-15 d after nadirs of drug induced neutropenia, as neutrophils were returning to the peripheral blood.

Separation and cryopreservation of bone marrow cells. Individual marrow specimens of 8-15 ml were withdrawn from the posterior iliac crest into syringes containing preservative-free heparin $(100 \mathrm{U} / \mathrm{ml}$, Gibco Laboratories, Grand Island, NY) and then diluted 1:12 (vol/vol) in calcium- and magnesium-free Dulbecco's PBS. Light-density marrow cells $(d<1.075)$ were then isolated by centrifugation on Percoll (Pharmacia Fine Chemicals, Uppsala, Sweden) gradients and were either studied directly in CFU-GM assays (see below) or were cryopreserved in $90 \%$ fetal bovine serum (FBS; Hyclone Sterile Systems, Logan, UT) and 10\% DMSO as described previously (17), in multiple aliquots $\left(5 \times 10^{7}\right.$ cells/aliquot $)$ such that replicate studies could be carried out with each marrow cell isolate.

Clonal growth of myeloid progenitor cells in the presence of rhGM$C S F$. Cryopreserved marrow cells were thawed, diluted in Iscove's modified Dulbecco's medium (IMDM, Gibco Laboratories), and washed twice by centrifugation ( $400 \mathrm{~g}$ for $5 \mathrm{~min}$ ) and resuspended in IMDM. These cells (or gradient-separated marrow cells that had not been cryopreserved) were then placed in $175-\mathrm{cm}^{2}$ tissue-culture flasks and incubated for $2 \mathrm{~h}$ at $37^{\circ} \mathrm{C}$. After incubation, cells that had not become adherent to flask surfaces were removed and placed into 35-mm-diameter tissue-culture dishes at a concentration of $1-5 \times 10^{4}$ cells/dish in IMDM supplemented with $0.8 \%$ methylcellulose $(4000$ $\mathrm{cp}$, Tridom/Fluke, Buchs, Switzerland), and 25\% FBS with or without rhGM-CSF (final concentration 0.1-2.0 nmol/liter). The final yield of nonadherent, light density-nucleated marrow cells that were recovered after density centrifugation, cryopreservation, thawing, and adherence depletion and were viable by due exclusion was routinely between 0.5 and $1.0 \times 10^{7}$ cells per $\mathrm{ml}$ of marrow aspirate originally drawn. These recoveries were the same for both normal volunteer and patient specimens. The purified rhGM-CSF used in these cultures was generously supplied to us by Dr. Gordon Wong of Genetics Institute, Cambridge, MA; its preparation has been described $(18,19)$. This rhGM-CSF preparation was $>97 \%$ pure and had a specific activity of 4.7 $\times 10^{6} \mathrm{U} / \mathrm{mg}$.

Myeloid (CFU-GM) colonies were counted with an inverted-phase microscope at a magnification of 40 at both 7 and $14 \mathrm{~d}$ of culture using standard scoring criteria $(17,20,21)$. Neutrophil, macrophage, and mixed neutrophil-macrophage colonies were also distinguished by clonal morphology and were counted separately. To verify the identity of colony types, individual colonies were plucked from the semi solid methylcellulose media of culture dishes, and the recovered cells were fixed and stained for direct morphologic examination. The growth of CFU-GM in response to varying concentrations of rhGM-CSF was examined on at least three separate occasions for each marrow cell isolate using replicate aliquots of cryopreserved marrow cells, and each individual study of marrow cells from patients was carried out together with a coincident study of marrow cells from at least two normal controls.

Analysis of rhGM-CSF dose-response studies. Estimates of the rhGM-CSF concentrations that elicited half-maximal growth of CFUGM in methylcellulose culture assays $\left(\mathrm{ED}_{\mathbf{5 0}}\right.$ 's) and their variances were calculated using a computer-assisted analytic approach based upon a modification of standard methods of probit analysis for quantal response data, described by Finney (22).

\section{Results}

Recovery of CFU-GM from the marrow of patients with cyclic neutropenia. None of the patients with childhood onset cyclic neutropenia were found to be deficient in myeloid progenitors. Rather, concentrations of CFU-GM in the marrow of these individuals were generally higher than normal, and the highest levels were found to coincide with nadirs of neutropenia during cycles. These findings, illustrated in Fig. 1, are consistent with prior reports (5-8). Data shown in this figure were obtained from CFU-GM assays in which cryopreserved marrow cells were cultured with $1.0 \mathrm{nmol} / \mathrm{liter}$ rhGM-CSF, a concentration of growth factor that was assumed to be well in excess of that required for maximum myeloid colony growth based upon the observations of others (19). Results similar to those shown in Fig. 1 were also obtained from CFU-GM assays of light-density, adherence-depleted marrow cells that had not been cryopreserved; note, however, that these marrow cell preparations were in all cases somewhat less enriched for CFU-GM (by a factor of 1.3-1.5) when compared with cryopreserved cells prepared from the same marrow aspirate speci-
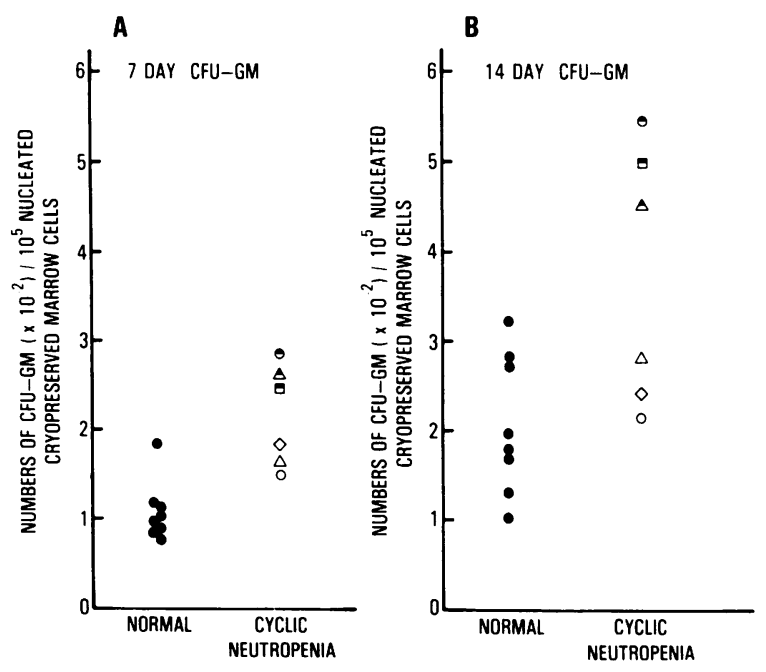

Figure 1. Concentrations of myeloid progenitors (CFU-GM) in cryopreserved marrow cell isolates. Each point represents a mean value derived from a minimum of three replicate studies with separate aliquots of eight individual marrow specimens obtained from eight normal volunteers and six marrow specimens from four patients with cyclic neutropenia. Marrow specimens were obtained twice from two cyclic neutropenic patients, once during the nadir (half-shaded symbols) and once during the recovery phase of cycles (open symbols); specimens were obtained from the other two patients either during a cycle nadir or during a recovery phase. 
men. The CFU-GM enrichment effect of cryopreservation was equivalent for marrow cells from both patients and normal controls, as were the recoveries of viable nucleated cells relative to the numbers of these cells in the original marrow aspirate specimens, after all isolation and cryopreservation procedures.

Under the culture conditions illustrated in Fig. 1, CFUGM of cyclic neutropenia patients and normal controls were indistinguishable both with respect to the relative numbers of neutrophil colonies (45-60\%), macrophage colonies (35-50\%), and mixed neutrophil-macrophage colonies (3-12\%) that developed during $14 \mathrm{~d}$ of culture and with respect to the morphology of these different types of colonies. The distinctive clonal morphologies of neutrophilic and macrophage colonies were verified by plucking individual colonies and examining the morphology of the cells recovered, after fixation and staining. As has been described by others $(20,21)$, neutrophilic colonies consisted of relatively small cells arrayed in a spread pattern around a variously cellular and compact center, whereas macrophage colonies, which consisted of relatively large, pleomorphic cells, were somewhat less cellular and less diffuse than neutrophilic colonies. Colonies that proved to be eosinophilic were also detected occasionally ( $<5 \%$ of total colonies) among CFU-GM grown with $\geq 1.0 \mathrm{nmol} /$ liter rhGMCSF. Whereas the clonal morphology of these colonies was somewhat similar to that of neutrophilic colonies, eosinophilic colonies were generally less cellular and more compact than neutrophilic colonies, and they consisted of cells that were more refractile when examined by phase microscopy. These rare colonies, which were present with an equivalent frequency in cultures of marrow cells from both patients and normal controls, were not included in CFU-GM counts.

Differences in the growth of CFU-GM with limiting concentrations of rhGM-CSF. Although there was no deficiency of CFU-GM in the marrow of patients with cyclic neutropenia, there were clear differences in the responses of CFU-GM to limiting concentrations of rhGM-CSF that distinguished these patients from controls, as shown in Fig. 2. In this figure, growth of CFU-GM at 7 and $14 \mathrm{~d}$ of culture in response to varying concentrations of rhGM-CSF is expressed as the percent of maximum colony growth observed with individual marrow cell isolates obtained from the four cyclic neutropenia patients, from two patients recovering from chemotherapy-induced myelosuppression, and from eight normal volunteers; whereas results of normal volunteer marrow studies have been combined to show mean values \pm 2 SD $(95 \%$ confidence limits), all data from the studies of marrow isolates from patients are shown separately.

As has been reported by others (19), maximum growth of CFU-GM from normal individuals was elicited by 20-100 pmol/liter rhGM-CSF. However, the dose responses for growth of CFU-GM from cyclic neutropenia marrow samples were uniformly shifted to the right, particularly for the 7-d CFU-GM, and $\geq 1.0 \mathrm{nmol} /$ liter rhGM-CSF was required to elicit maximal clonal proliferation of these progenitors. These differences in the rhGM-CSF responsive growth of CFU-GM were equally apparent with marrow cells obtained during nadirs and recovery phases of cycles and with various cell plating concentrations in the clonal assays. Results shown in Fig. 2 were obtained with marrow cell plating concentrations of $3 \times 10^{4}$ cells/plate; very similar results were obtained with plating concentrations of both 1 and $5 \times 10^{4}$ cells/plate in
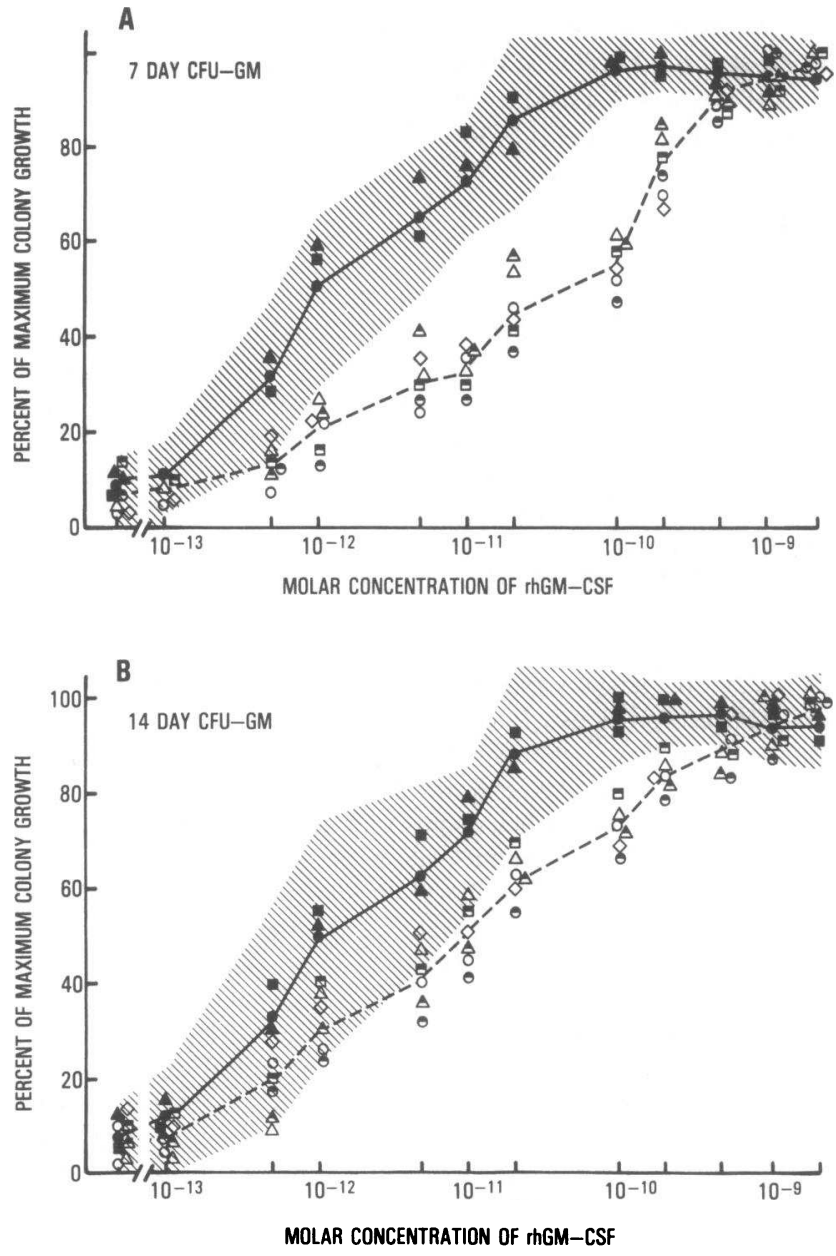

Figure 2. Growth of CFU-GM at $(A) 7$ and $(B) 14 \mathrm{~d}$ with varying concentrations of rhGM-CSF (0.1-2.0 nmol/liter). Results are expressed as percents of maximum colony growth observed within this dose range with the separate marrow cell isolates. $\bullet, \ldots$, mean values for marrow cells from eight normal volunteers and the shaded areas depict 2 SD above and below these mean values (95\% confidence interval). $\Delta, a$, results from two patients recovering from drug-induced myelosuppression. The open and half-shaded symbols represent results for six marrow specimens from patients with cyclic neutropenia, obtained during the recovery phase or nadir of cycles, respectively; - - -, mean values for the growth of CFU-GM from these patients.

separate studies. In contrast, the rhGM-CSF responsive growth of myeloid progenitor cells from patients recovering from drug-induced myelosuppression was not different from normal. Marrow cell isolates from these individuals were enriched in CFU-GM (420-540 CFU-GM [14 d]/105 cryopreserved marrow cells) and were derived from marrow aspirate specimens that histologically showed a substantial increase in relative numbers of immature neutrophilic forms (promyelocytes and myelocytes), like the marrow specimens of cyclic neutropenia patients during and immediately after neutropenic nadirs.

Because differences in rhGM-CSF responses were most apparent with the predominantly neutrophilic $(>80 \%) 7$ day CFU-GM of cyclic neutropenia patients, it was of interest to analyze the rhGM-CSF responsive growth of 14-d neutrophilic and macrophage colonies separately. Table I presents mean 
Table I. ED $5 D^{\prime}$ 's of rhGM-CSF for Stimulation of CFU-GM Growth

\begin{tabular}{ccccc}
\hline & & \multicolumn{2}{c}{ Cyclic neutropenia } & \\
\cline { 3 - 4 } & Normal & Nadir & Recovery & \\
\hline & & & & \\
7-d CFU-GM & $2.2 \pm 0.5(8)^{*}$ & $31.6 \pm 8.1(3)$ & $34.3 \pm 5.8(3)$ & $P<0.001$ \\
14-d CFU-GM & $1.6 \pm 0.4(8)$ & $7.4 \pm 1.6(3)$ & $6.9 \pm 2.2(3)$ & $P<0.01$ \\
14-d CFU-GM & & & & \\
(neutrophilic) & $5.1 \pm 1.3(8)$ & $27.5 \pm 6.6(3)$ & $30.7 \pm 7.8(3)$ & $P<0.001$ \\
$\begin{array}{c}\text { 14-d CFU-GM } \\
\text { (macrophage) }\end{array}$ & $0.7 \pm 0.2(8)$ & $0.9 \pm 0.6(3)$ & $1.3 \pm 0.7(3)$ & NS
\end{tabular}

* Concentration of rhGM-CSF in picomoles per liter required for half-maximal growth of CFU-GM; results represent mean values \pm SEM (number of separate marrow aspirate specimens examined); the eight aspirate specimens from normal volunteers and 6 aspirate specimens from cyclic neutropenia patients are the same as those described in Fig. 1 and 2; $P$ values for statistically significant differences between normal and cyclic neutropenia marrow cell isolates by $t$ test are shown.

values for the concentrations of rhGM-CSF $\left(\mathrm{ED}_{50}\right)$ that stimulated half-maximal growth of CFU-GM. These values were calculated from computer-fitted curves applied to dose-response data derived from replicate studies. As could be anticipated from the data shown in Fig. 2, the mean $\mathrm{ED}_{50}$ of rhGMCSF required for growth of 7-d CFU-GM from cyclic neutropenia patients was substantially higher than that observed with marrow cells from normal volunteers. $\mathrm{ED}_{50}$ 's required for growth of 14-d CFU-GM were also significantly higher than normal, although absolute differences were less. However, when 14-d neutrophilic and macrophage colonies were analyzed separately, it was evident not only that maximal and half-maximal growth of macrophage colonies required less GM-CSF than that required for growth of neutrophilic colonies, as has been reported by others (21), but also that the aberrant rhGM-CSF responsive growth of CFU-GM from cyclic neutropenia patients reflected primarily, if not entirely, an abnormality in the growth of neutrophilic colonies.

Results shown in Fig. 2 and Table I are from studies of cryopreserved marrow cell isolates, each of which was examined repeatedly. However, the abnormalities of GM-CSF responsive growth of myeloid progenitors from cyclic neutropenia patients that are shown were equally apparent in separate studies of noncryopreserved marrow cell isolates. In a representative study, in which cryopreserved and noncryopreserved cells from the same marrow aspirate specimens were studied in parallel, the $\mathrm{ED}_{50}$ 's of rhGM-CSF for stimulating 7-d CFU-GM growth were found to be 2.48 and $2.61 \mathrm{pmol} /$ liter, respectively, for cryopreserved and noncryopreserved cells from an individual normal donor marrow aspirate specimen, vs. 24.9 and $18.3 \mathrm{pmol} /$ liter for cryopreserved and noncryopreserved cells from a cyclic neutropenia marrow specimen; corresponding $\mathrm{ED}_{50}$ 's for 14-d CFU-GM growth were 1.68 and $1.38 \mathrm{pmol} /$ liter for the normal marrow cells and 8.32 and $9.93 \mathrm{pmol} / \mathrm{liter}$ for the cyclic neutropenia marrow cells.

\section{Discussion}

Results of these studies indicate that the numbers of myeloid progenitor cells (CFU-GM) recoverable from the marrow of patients with childhood onset cyclic neutropenia are normal or increased. This finding is consistent with earlier reports (5-8). However, our studies also find that dose-response curves for the rhGM-CSF-stimulated clonal proliferation of CFU-GM from these patients are shifted to the right. This abnormality in rhGM-CSF responsive growth of CFU-GM in cyclic neutropenia is independent of cycle time; it cannot be eliminated (or reproduced in normal marrow cells) by varying cell plating concentrations in clonal assays, nor is it evident in marrow cells obtained from patients during recovery from profound drug-induced myelosuppression when concentrations of CFU-GM in the marow are substantially expanded. Furthermore, abnormalities in the rhGM-CSF responsive growth of CFU-GM in cyclic neutropenia are found primarily to affect the growth of progenitor cells committed to neutrophilic differentiation. This latter finding is particularly noteworthy because it matches the clinical characteristics of dysregulated myelopoiesis in this disease. Whereas neutrophil production is markedly impaired in cyclic neutropenia, monocyte production is typically maintained at normal to increased levels.

Our findings are consistent with the conclusion that childhood onset cyclic neutropenia is associated with an intrinsic disturbance in the responsiveness of myeloid progenitor cells to GM-CSF. This conclusion is of particular interest in that mathematical models of the regulation of myelopoiesis, based upon the distinctive characteristics of cyclic neutropenia, have predicted that an abnormality in the responsiveness of progenitor cells to regulatory signals could produce the oscillations in blood cell production that are observed in this disease (12). However, our findings must be interpreted with caution in this regard.

Although varying concentrations of pure, rhGM-CSF constituted the sole experimental variable in progenitor cell assays (other than the source of progenitor cells), it is unlikely that the expression of myeloid colony growth depended only upon the presence of this growth factor in the in vitro culture system that was used. There is compelling evidence from recent studies that clonal proliferation and differentiation of hematopoietic progenitor cells are modulated by and may require the cooperation of multiple humoral signals $(21,23-26)$. Hence, abnormalities in the in vitro growth of myeloid progenitor cells from cyclic neutropenia patients could reflect a disturbance in the responsiveness of progenitor cells to a separate, cooperative signal provided by the FBS present in culture media or generated by residual accessory cells in marrow cell isolates. Indeed, the lineage-restricted character of the abnormalities in rhGM-CSF responsive progenitor cell growth that were observed suggests that a separate regulatory factor more specific to the neutrophilic lineage (such as G-CSF) might also be involved.

Additional studies with more highly purified preparations of hematopoietic stem cells and with defined, serum-free culture conditions will be required to further characterize the abnormal responses of myeloid progenitors to GM-CSF in cyclic neutropenia that are revealed when limiting concentrations of this growth factor are examined in detail. Nonetheless, these studies provide an experimental basis for considering the use of exogenously administered recombinant myelopoietic growth factors to treat patients with childhood onset cyclic neutropenia, for such treatment might overcome or compensate for the disturbance of GM-CSF responsive myeloid progenitor cell growth that is evident in this disease. 


\section{Acknowledgments}

The authors gratefully acknowledge the help and advice of Dr. Elizabeth $\mathrm{C}$. Wright in the computer-assisted analysis of results.

\section{References}

1. Wright, D. G., D. C. Dale, A. S. Fauci, and S. M. Wolff. 1981. Human cyclic neutropenia: clinical review and long-term follow-up of patients. Medicine (Baltimore). 60:1-13.

2. Loughran, T. P., E. A. Clark, T. H. Price, and W. P. Hammond. 1986. Adult onset cyclic neutropenia is associated with increased large granular lymphocytes. Blood. 68:1082-1087.

3. Wright, D. G., A. S. Fauci, D. C. Dale, and S. M. Wolff. 1978. Correction of human cyclic neutropenia with prednisolone. $N$. Engl. J. Med. 298:295-300.

4. Guerry, D., D. C. Dale, M. Omine, S. Perry, and S. M. Wolff. 1973. Periodic hematopoiesis in human cyclic neutropenia. J. Clin. Invest. 52:3220-3230.

5. Dresch, C., D. Thevenian, H. Castro-Malaspina, and A. Faille. 1977. Cell kinetics in human cyclic neutropenia. Scand. J. Haematol. 19:14-24.

6. Jacobsen, N., and H. E. Broxmeyer. 1979. Oscillations of granulocyte and megakaryocyte progenitor cell populations in cyclic neutropenia in man. Scand. J. Haematol. 23:33-36.

7. Greenberg, P. L., I. Bax, J. Levin, and T. H. Andrews. 1979. Alteration of colony stimulating factor output, endotoxemia, and granulopoiesis in cyclic neutropenia. Am. J. Hematol. 1:375-385.

8. Mizoguchi, H., S. Chyoda, Y. Miura, F. Takabu, S. Asano, S. Suzuki, A. Urabe, S. Chiba, K. Kosaka, and S. Miwa. 1976. Colony forming cells in culture and colony stimulating activity in the urine and the serum in a case of cyclic neutropenia. Tohoku J. Exp. Med. 119:317-324.

9. King-Smith, E. A., and A. Morley. 1970. Computer simulation of granulopoiesis: normal and impaired granulopoiesis. Blood. $36: 254-262$.

10. Rubinow, S. I., and J. L. Lebowitz. 1975. A mathematical model of neutrophil production and control in normal man. J. Math. Biol. 1:187-225.

11. Mackey, M. C. 1978. Unified hypothesis for the origin of aplastic anemia and periodic hematopoiesis. Blood. 51:941-956.

12. Von Sculthess, G. K., and N. A. Mazer. 1982. Cyclic neutropenia: a clue to the control of granulopoiesis. Blood. 59:27-37.

13. Metcalf, D. 1986. The molecular biology and functions of the granulocyte-macrophage colony stimulating factors. Blood. 67:257-267.
14. Clark, S. C., and R. Kamen. 1987. The human hematopoietic colony stimulating factors. Science (Wash. DC). 236:1229-1237.

15. Moore, M. A. S. 1984. Humoral regulation of granulopoiesis. Clin. Hematol. 8:287-309.

16. Broxmeyer, H. E., M. DeSousa, A. Smithyman, P. Ralph, J. Hamilton, J. I. Kurland, and J. Bognacki. 1980. Specificity and modulation of the action of lactoferrin, a negative feedback regulator of myelopoiesis. Blood. 55:324-333.

17. Meagher, R. C., A. J. Salvado, and D. G. Wright. 1988. An analysis of the multilineage production of human hematopoietic progenitors in long-term bone marrow culture: evidence that reactive oxygen intermediates derived from mature phagocytic cells have a role in limiting progenitor cell self-renewal. Blood. 72:273-281.

18. Wong, G. C., J. S. Witek, P. A. Temple, K. M. Wilkens, A. C. Leary, D. P. Luxemberg, S. S. Jones, E. L. Brown, R. M. Kay, E. C. Orr, C. Shoemaker, D. W. Golde, R. J. Kaufman, R. M. Hewick, E. A. Wang, and S. C. Clark. 1985. Human GM-CSF: molecular cloning of complementary DNA and purification of the natural and recombinant proteins. Science (Wash. DC). 228:810-815.

19. Gasson, J. C., S. E. Kaufman, R. H. Weisbart, M. Tomonaga, and D. W. Golde. 1986. High affinity binding of granulocyte-macrophage colony-stimulating factor to normal and leukemic human myeloid cells. Proc. Natl. Acad. Sci. USA. 83:669-673.

20. Iscove, N. M., F. Sieber, and K. H. Winterhalter. 1974. Erythroid colony formation in cultures of mouse and human bone marrow. J. Cell. Physiol. 83:309-320.

21. Walker, F., N. A. Nicola, D. Metcalf, and A. W. Burgess. 1985. Hierarchical down regulation of hemopoietic growth factor receptors. Cell. 43:269-276.

22. Finney, D. J. 1971. Probit Analysis. 3rd ed. Oxford University Press, New York. 203-205.

23. Leary, A. G., K. Ikebuchi, Y. Hirai, G. C. Wong, Y.-C. Yang, S. C. Clark, and M. Ogawa. 1988. Synergism between interleukin-6 and interleukin-3 in supporting proliferation of human hematopoietic stem cells: comparison with interleukin- $1 \alpha$. Blood. 71:1759-1763.

24. Miyajima, A., S. Miyatake, J. Schreurs, J. DeVries, N. Arai, T. Yokota, and K. Arai. 1988. Coordinate regulation of immune and inflammatory responses by T-cell derived lymphokines. FASEB (Fed. Am. Soc. Exp. Biol.) J. 2:2462-2473.

25. Sieff, C. A., S. Ekern, and D. G. Nathan. 1988. Combinations of purified recombinant colony stimulating factors are required for optimal hematopoietic differentiation. Clin. Res. 36:615a.(Abstr.)

26. Migliaccio, G., A. R. Migliaccio, and J. W. Adamson. 1988. In vitro differentiation of human granulocyte/macrophage and erythroid progenitors: comparative analysis of the influence of recombinant human erythropoietin, G-CSF, GM-CSF, and IL-3 in serum-supplemented and serum-deprived cultures. Blood. 72:248-256. 